\title{
Crecimiento estacional y rentabilidad de plantaciones forestales comerciales de pino radiata en suelos de trumao según método de establecimiento*
}

\author{
Seasonal growth and yield of radiata pine commercial forest plantations on volcanic ("trumao") soils.
}

\author{
MANUEL TORAL I., ANGELO FRATTI B., LUIS A. GONZALEZ R.
}

E-mail: mtoral@uchile.cl

\begin{abstract}
SUMMARY
In 1994, Forestal Mininco started a research project to assess the effects of different establishment methods on the seasonal growth and yield of radiata pine (Pinus radiata, D. Don) plantations. Many survey trials were set up during that year using coastal metamorphic, volcanic "trumao", sandy, and granitic soils. Based on the above, this article focuses on the edafoclimatic characteristics of volcanic "trumao" soils. With this general consideration, three approaches were defined -a Basic or Traditional Method (MB), an Improved Method (MM), and an Optimum Method (MO)- to improve the plantation establishment, initial plant growth development, and to assess the plantation yield corresponding to each method. In each experiment, tree measurements were recorded over a six-year period. Associated climatic and economic information was also obtained. After analyzing the results, it was concluded that these establishment methods have no effect on the seasonal growth of radiata pine. However, in "trumao" soil conditions, the Productivity Index $\left(\mathrm{DAC}^{2} * \mathrm{H}\right)$ showed two growth peaks: one occurring at the beginning of summer and the other in the middle of the fall. Statistical analysis of the methods determined that, although they had no significant effect over tree diameter and growth height, they do have an effect on the Productivity Index $\left(\mathrm{DAC}^{2} * \mathrm{H}\right)$. Given the above, the Basic Method applied on "trumao" soils was assessed economically. The results indicate that plantations established on these soils give maximum yields of the products considered in the economic evaluation when harvested at an age of 21 years.
\end{abstract}

Keywords: silviculture, plantations, radiata pine.

\section{RESUMEN}

En el año 1994, Forestal Mininco S. A. estableció un proyecto de investigación que tuvo como objetivo estudiar el efecto de métodos de establecimiento sobre el crecimiento estacional y rentabilidad de dichos métodos en plantaciones comerciales de Pinus radiata D. Don. Para tales efectos se establecieron en 1994 diversos ensayos de establecimiento en suelos metamórficos costeros, suelos de trumao, suelos de arena y en suelos graníticos. Dado lo anterior, en este artículo sólo se analiza la condición edafoclimática suelo de "Trumao". En esta condición, se definieron tres tratamientos de plantación: Método Básico o Tradicional (MB), Método Mejorado (MM) y Método Optimo (MO), con el objeto de mejorar el prendimiento y desarrollo inicial de la plantación, así como evaluar la rentabilidad según tratamiento aplicado. Para realizar el presente estudio se recopilaron durante 6 años (1994-2000) los antecedentes dasométricos de los ensayos, además de procesar los correspondientes datos climáticos y económicos de dichos ensayos. El análisis de la información demostró, en primer lugar, que los métodos de establecimiento tienen poca o nula influencia sobre el crecimiento estacional de pino radiata. En la condición suelos de "Trumao", el índice de productividad $\left(\mathrm{DAC}^{2} * \mathrm{H}\right)$ presentó dos puntos de máximo crecimiento: uno a principios de verano y el otro a mediados de otoño. El análisis estadístico determinó que los métodos de establecimiento no tienen efecto significativo sobre el crecimiento en diámetro y altura del árbol. Sí lo tienen en el índice de productividad $\mathrm{DAC}^{2} * \mathrm{H}$. Dado lo anterior se evaluó econonómicamente sólo el método básico de los suelos de "Trumao". Los resultados de esta evaluación demuestran que las plantaciones en dichos suelos son rentables, definiéndose una edad de cosecha de 21 años para los productos analizados.

Palabras clave: silvicultura, plantaciones, pino radiata.

Se agradece a los ingenieros forestales señores: Jaime Salas A. y José Alvarez M., de la Empresa Forestal Mininco, por la colaboración prestada para elaborar este trabajo. A ellos, y a la Empresa, "Muchas Gracias". 


\section{INTRODUCCION}

En Chile, hace 50 años, la inversión y las técnicas de establecimiento de plantaciones forestales comerciales eran mínimas, lo que provocó que las plantaciones tuvieran bajos prendimientos y escaso crecimiento, lo que obligaba a replantar grandes superficies para tener un bosque comercial.

Dado lo anterior, se decidió dirigir parte de los recursos gastados en los replantes en mejorar las técnicas y métodos de plantación. Estas técnicas se conocen actualmente como protocolos de plantación y tratan de controlar los factores más limitantes del establecimiento y crecimiento inicial de una plantación. Estos factores son: el suelo (estructura, nutrición, profundidad entre otros), disponibilidad de agua y calidad de la planta (calidad genética y morfología).

Desde que se promulgó, en 1974, el Decreto Ley $\mathrm{N}^{\circ} 701$, sobre fomento forestal, las plantaciones forestales comerciales de pino radiata en Chile han aumentado notablemente, llegando a alcanzar en el año 2004 una superficie aproximada de 2,1 millones de hectáreas, establecidas en distintas condiciones edafoclimáticas.

Esta superficie plantea la necesidad de desarrollar proyectos de investigación que tengan como fin mejorar la rentabilidad de las plantaciones con distintas condiciones de suelo, clima y métodos de establecimiento.

Dado lo anterior, la hipótesis de este trabajo es que la utilización de una silvicultura integrada e intensiva en una determinada condición edafoclimática influye sobre la sobrevivencia, crecimiento inicial y rentabilidad de un cultivo forestal de pino. Por lo tanto, se pretende determinar el nivel de inversión en el establecimiento de las plantaciones que asegure la máxima rentabilidad según condición de clima y suelo, además de determinar la curva de crecimiento estacional con el fin de estudiar la dinámica de crecimiento de la especie a lo largo del año. Esta última información puede ser utilizada como una herramienta en el proceso de toma de decisiones sobre la ocurrencia de actividades como: aplicación de herbicidas, desbroces, fertilizaciones, podas, raleos, inventarios, entre otras actividades.

El objetivo general de este estudio es evaluar el crecimiento estacional y la rentabilidad de Pinus radiata $\mathrm{D}$. Don, utilizando tres métodos de establecimiento de plantaciones, para la condición de suelos de "Trumao". Para lo cual se presentan los siguientes objetivos específicos:

- Determinar las curvas de crecimiento acumulado y estacional para el diámetro a la altura del cuello (DAC), altura total de la planta $(\mathrm{H})$ $y$ el índice de productividad $\left(\mathrm{DAC}^{2 *} \mathrm{H}\right)$.

- Determinar para los suelos de "Trumao" si el crecimiento de las variables mencionadas en Pinus radiata difiere estadísticamente, según el método de establecimiento utilizado.

- Evaluar la rentabilidad de Pinus radiata, según método de establecimiento.

Las prácticas más utilizadas en el establecimiento de pino radiata son el subsolado, control de malezas pre y postplantación y fertilización de apoyo al establecimiento de la plantación. El subsolado persigue mejorar las características estructurales del suelo para acrecentar el desarrollo de las raíces y facilitar el drenaje. El control de malezas persigue eliminar o disminuir la competencia, en especial agua y nutrientes, entre las plantas jóvenes de pino con especies no deseables; y la fertilización, incrementar la nutrición de la especie y que ésta tenga un rápido desarrollo inicial.

En un estudio realizado en Sudáfrica se concluyó que para maximizar los beneficios económicos y ecológicos de una plantación de pino radiata de cuatro años se deben utilizar en el establecimiento plantas con un diámetro de cuello promedio de 4,1 mm y un límite máximo de malezas de $1.500 \mathrm{~kg}$ de biomasa por hectárea, mientras que los tratamientos al suelo no se justifican en términos económicos (1).

A su vez, Mason et al. (2) determinaron que para el establecimiento de pino radiata era necesario utilizar la mejor calidad de planta y un esquema de control de malezas, para maximizar el desarrollo de la misma en los primeros años de vida. Al mismo tiempo, concluyeron que los tratamientos al suelo (subsolado) eran más recomendables para suelos húmedos y mal drenados, que para suelos ligeros y bien drenados.

Por otro lado, Balneaves et al. (3), en un estudio realizado sobre un suelo seco de Canterbury, Nueva Zelandia, concluyeron que la calidad de la planta producida en vivero, el manejo de ella postvivero (poda de raíces a $10 \mathrm{~cm}$, almacenamiento en cuarto frío y plantación en no más de 24 horas), el subsolado, y principalmente el control de male- 
zas, son los factores más significativos en el incremento de volumen de la especie a largo plazo.

En la región costera de Oregón, Estados Unidos, se realizó un estudio que duró siete años, en el cual se determinó la sobrevivencia y el crecimiento de Pseudotsuga menziessi; aplicando seis métodos de preparación del sitio. Los resultados del estudio concluyeron que la sobrevivencia, el diámetro del tallo a $15 \mathrm{~cm}$ sobre el suelo y la altura fueron superiores al utilizar métodos de quema y aplicación de herbicidas, que al utilizar un método manual de limpia al momento de plantar o no utilizar ningún método de establecimiento (4).

De igual forma, Duryea y Dougherty (5), como Maclaren (6), establecen que los suelos que regularmente necesitan del subsolado son aquellos con presencia de horizontes arcillosos y de grava, muy compactados y de alta densidad aparente $\left(1,4-1,8 \mathrm{~g} / \mathrm{cm}^{3}\right)$. Por otro lado, este subsolado debe realizarse sobre suelos secos y en forma paralela a las curvas de nivel del terreno para evitar erosión, además se puede considerar un control previo de malezas.

Por otra parte, Jackson et al. (7) señalan que la precipitación está directamente relacionada con el crecimiento de pino radiata en Nueva Zelandia, donde el crecimiento en altura alcanza dos "peaks" importantes, el primero en los meses de octubrenoviembre (primavera) y el segundo en los meses de marzo-abril (principios de otoño). Por otra parte, el crecimiento en diámetro alcanza su máximo valor en febrero (finales del verano).

La temperatura es también un factor ambiental importante, que, de acuerdo con Gutiérrez (8), influye sobre la actividad de los meristemas apicales, los cuales cesan su actividad cuando la temperatura desciende a 5 ó $6^{\circ} \mathrm{C}$. Por otra parte, Tennent (9) y Burdon (10) establecen que la especie tiene distintos patrones de crecimiento a medida que varía el sitio; tal es así que el crecimiento en diámetro no se comporta de la misma manera que el crecimiento en altura.

\section{MATERIAL Y METODOS}

La condición estudiada corresponde al predio Santa Lucía, ubicado cerca de Trilahue, precordillera andina, donde predominan los suelos de "Trumao", los cuales están clasificados como Ashy, medial, mesic Typic Dystrandept, y con material parental compuesto por cenizas volcánicas recientes.

Los suelos de "Trumao" (palabra mapuche que significa suelo polvoriento en verano) son profundos $(120 \mathrm{~cm})$, de texturas finas a medias y no presentan variación a lo largo del perfil. Los colores están dentro del $10 \mathrm{YR}$, de topografías variables. En general, estos suelos poseen excelentes cualidades físicas para su cultivo, tales como alto contenido de materia orgánica, buena estructura y permeabilidad, siendo su principal limitante la fijación de fósforo; además, tienen una baja densidad aparente.

\section{DESCRIPCION DE LOS METODOS DE ESTABLECIMIENTO}

Se ensayaron tres métodos de establecimiento que se exponen en el cuadro 1.

En otras palabras, los métodos de establecimiento difieren básicamente en la intensidad de preparación del suelo y en la fertilización de apoyo al establecimiento.

A continuación se presenta un listado de las variables utilizadas y métodos generales para la realización de este trabajo:

- Variables de estado medidas: diámetro altura de cuello (DAC), altura $(\mathrm{H})$, número de árboles, diámetro altura de pecho (DAP) y el índice de productividad, dado por la expresión: $\mathrm{DAC}^{2 *} \mathrm{H}$. Las variables DAC y $\mathrm{H}$ fueron medidas cada dos meses a partir del establecimiento hasta que los rodales alcanzaron los cuatro años y medio de edad. El DAP fue medido a partir de los tres años de edad. El DAC y el DAP se midieron colocando una marca en cada árbol a 0,3 y 1,3 m de altura respectivamente, a objeto de medir permanentemente en el mismo punto. Para los diámetros se utilizó un pie de metro con precisión al milímetro, mientras que la altura se midió con una varilla graduada al centímetro, colocada al lado del árbol.

- Se caracterizó el suelo y se midieron mensualmente las temperaturas y precipitación para los seis años que duró el estudio.

- La variable económica incluye los costos actuales de todas las actividades silviculturales a lo largo del período de estudio y los precios de los productos obtenidos. 


\section{CUADRO 1}

Método de establecimiento en suelos de Trumao.

Establishment method on "trumao" soils.

\begin{tabular}{|c|c|c|c|}
\hline $\begin{array}{l}\text { Actividades } \\
\text { Realizadas }\end{array}$ & $\begin{array}{l}\text { Básico } \\
\text { MB }\end{array}$ & $\begin{array}{l}\text { Mejorado } \\
\text { MM }\end{array}$ & $\begin{array}{l}\text { Óptimo } \\
\text { MO }\end{array}$ \\
\hline $\begin{array}{l}\text { Control de maleza } \\
\text { preplantación }\end{array}$ & Roundup (4 1/ha) & Roundup (4 1/ha) & Roundup (4 1/ha) \\
\hline Preparación del suelo & Casilla $\left(15^{*} 15^{*} 12 \mathrm{~cm}\right)$ & Casilla $(27 * 27 * 27 \mathrm{~cm})$ & Casilla $(30 * 30 * 30 \mathrm{~cm})$ \\
\hline $\begin{array}{l}\text { Baño fosfatado de } \\
\text { la raíz de la planta }\end{array}$ & - & - & SFT (4 kg/200 litros) \\
\hline Plantación & Manual & Manual & Manual \\
\hline Fecha & Finales de otoño 1994 & Finales de otoño 1994 & Finales de otoño 1994 \\
\hline Técnica & $2 \mathrm{~T}$ & $4 \mathrm{~T}$ & $4 \mathrm{~T}$ \\
\hline Distanciamiento (m) & $3,5 \times 2$ & $3,5 \times 2$ & $3,5 \times 2$ \\
\hline Especie & Pino radiata & Pino radiata & Pino radiata \\
\hline Origen & Cutting (raíz desnuda) & Cutting (raíz desnuda) & Cutting (raíz desnuda) \\
\hline $\begin{array}{l}\text { Control de maleza } \\
\text { postplantación }\end{array}$ & $\begin{array}{l}\text { Galant }(1 \mathrm{~kg} / \mathrm{ha}) \\
\text { Simazina }(3 \mathrm{~kg} / \mathrm{ha})\end{array}$ & $\begin{array}{l}\text { Galant }(1 \mathrm{~kg} / \mathrm{ha}) \\
\text { Simazina }(3 \mathrm{~kg} / \mathrm{ha})\end{array}$ & $\begin{array}{l}\text { Galant }(1 \mathrm{~kg} / \mathrm{ha}) \\
\text { Simazina }(3 \mathrm{~kg} / \mathrm{ha})\end{array}$ \\
\hline Fertilización & Operacional & Operacional & $\begin{array}{l}\mathrm{N}, \mathrm{P}, \mathrm{K}, \mathrm{Ca}, \mathrm{Mg}, \mathrm{Mn}, \mathrm{Fe}, \\
\mathrm{Cu}, \mathrm{Zn}, \mathrm{B}\end{array}$ \\
\hline
\end{tabular}

Fuente: Forestal Mininco S.A. Proyecto Silvicultura Integrada de Plantaciones 2001 (11).

Notas: La fertilización para el MO se aplicó durante la primera quincena de noviembre de 1994, en dos medias lunas a $15 \mathrm{~cm}$ de la planta y a una profundidad de 5 a $10 \mathrm{~cm}$ mezclado con el suelo. Se aplicaron $30 \mathrm{~g} / \mathrm{planta}$ de la mezcla de fertilizante propuesta a base del análisis químico del suelo, la cual aportó las siguientes cantidades en gramos por ingrediente activo por planta: N: 5,2-P: 11,4-K: 0,3-Mn: 0,2-Zn: 0,2-Fe: 1,8-Ca: 3,3-Cu: 0,3-Mg: 1,2-B: 0,8. La fertilización operacional consiste en la aplicación de $175 \mathrm{~g} /$ planta de una mezcla comercial del tipo N-P-K con la siguiente formulación: 14-15-9, la forma de aplicación fue la misma explicada anteriormente.

- Programa estadístico SAS®, para el análisis estadístico de los datos.

- Acceso al simulador de crecimiento de pino radiata D. Don, Radiata Plus versión 5,2, propiedad de la empresa Forestal Mininco S.A.

Para cumplir con los objetivos de este trabajo es necesario el análisis de la información desde tres puntos de vista. El análisis de crecimiento estacional y su relación con variables climáticas, el análisis estadístico de las variables de crecimiento y el análisis económico de los ensayos efectuados.

El análisis de crecimiento estacional y su relación con las variables climáticas, y el análisis estadístico de las variables de crecimiento se rea- lizó con el fin de determinar la curva de crecimiento estacional del índice de productividad, y para determinar si el crecimiento de pino radiata difiere según método de establecimiento. Para cumplir con lo anterior, cada método de establecimiento constó con tres parcelas permanentes de medición compuestas de 40 árboles cada una, es decir, 120 árboles por tratamiento. Al mismo tiempo, los ensayos descritos se instalaron bajo un diseño estadístico completamente al azar de efectos fijos, registrando las variables de estado cada dos meses y durante seis años.

El análisis económico se realizó mediante los indicadores de rentabilidad: Valor Actual Neto (VAN), Valor Potencial del Suelo (VPS) y la Tasa Interna de Retorno (TIR), todos a una tasa de ac- 
tualización del 8\%-10\% y $12 \%$. Sin embargo, hay que destacar que previo al análisis económico cada variable dasométrica de los ensayos efectuados se proyectó a la edad de cosecha mediante el simulador de crecimiento, manejo y trozado Radiata Plus versión 5,2 de propiedad de Forestal Mininco S.A.

\section{RESULTADOS Y CONCLUSIONES}

ANALISIS DEL CRECIMIENTO ESTACIONAL EN LA ZONA EDAFOCLIMATICA DE SUELOS DE “ TRUMAO”

\section{- Diagrama Ombrotérmico de Gaussen-Walter}

En la figura 1 se presenta el diagrama ombrotérmico del fundo Santa Lucía y en la figura 2, las curvas de crecimiento acumulado y estacional para el índice de productividad $\mathrm{DAC}^{2 *} \mathrm{H}$.

La figura 1 muestra las variaciones mensuales en temperatura a lo largo del año. Estas variaciones indican que las temperaturas más altas se alcanzan entre los meses de diciembre y febrero, mientras que las más bajas se registran constantemente entre los meses de junio a agosto. Por otra parte, las variaciones mensuales en precipitación son más irregulares, siendo junio, julio y agosto los meses del año con mayor precipitación; en contraste, los meses de diciembre, enero, febrero y marzo presentan las menores cantidades de agua caída. Además, el diagrama anterior sirve para identificar a enero, febrero y marzo como los meses secos del año para esta condición edafoclimática.

- Curvas de crecimiento estacional y acumulado para el índice de productividad $\mathrm{DAC}^{2 *} \mathrm{H}$

En las figuras 2 y 3 se puede observar que la variable $\mathrm{DAC}^{2 * \mathrm{H}}$, para todos los tratamientos, muestra sus valores más altos en los meses de marzo, abril y mayo (otoño), mientras que el menor crecimiento estacional ocurre entre julio y agosto (invierno). Para el crecimiento acumulado, este índice no muestra diferencias entre los tres tratamientos empleados y el rango de mayor crecimiento está con precipitaciones por encima de los $100 \mathrm{~mm}$ y temperaturas próximas a $\operatorname{los} 12^{\circ} \mathrm{C}$, y el menor crecimiento durante el invierno.

- Curvas de crecimiento estacional y acumulado para las variables altura y diámetro a la altura del cuello (DAC)

En la figura 4 se presenta la curva de crecimiento estacional y acumulado para la variable altura.

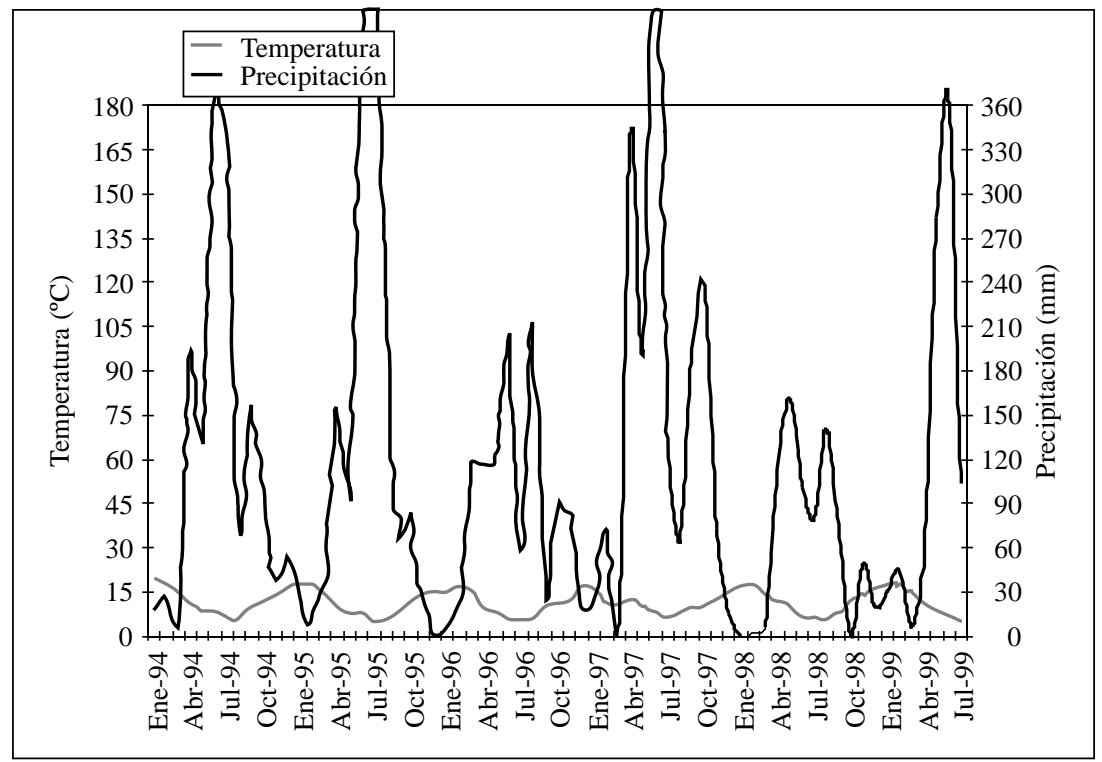

Figura 1. Diagrama ombrotérmico de Gaussen.- Walter.

Gaussen-Walter ombrothermic diagram. 


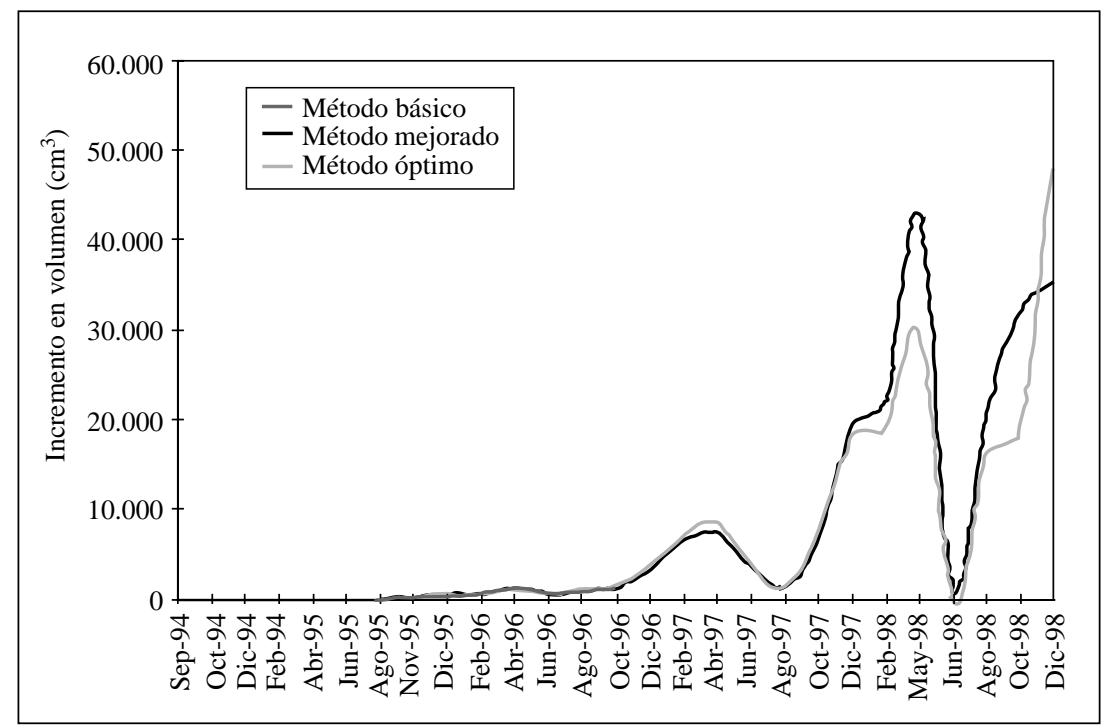

Figura 2. Curva de crecimiento estacional para el índice $\mathrm{DAC}^{2 *} \mathrm{H}$. Seasonal $\mathrm{DAC}^{2} * \mathrm{H}$ index growth curve.

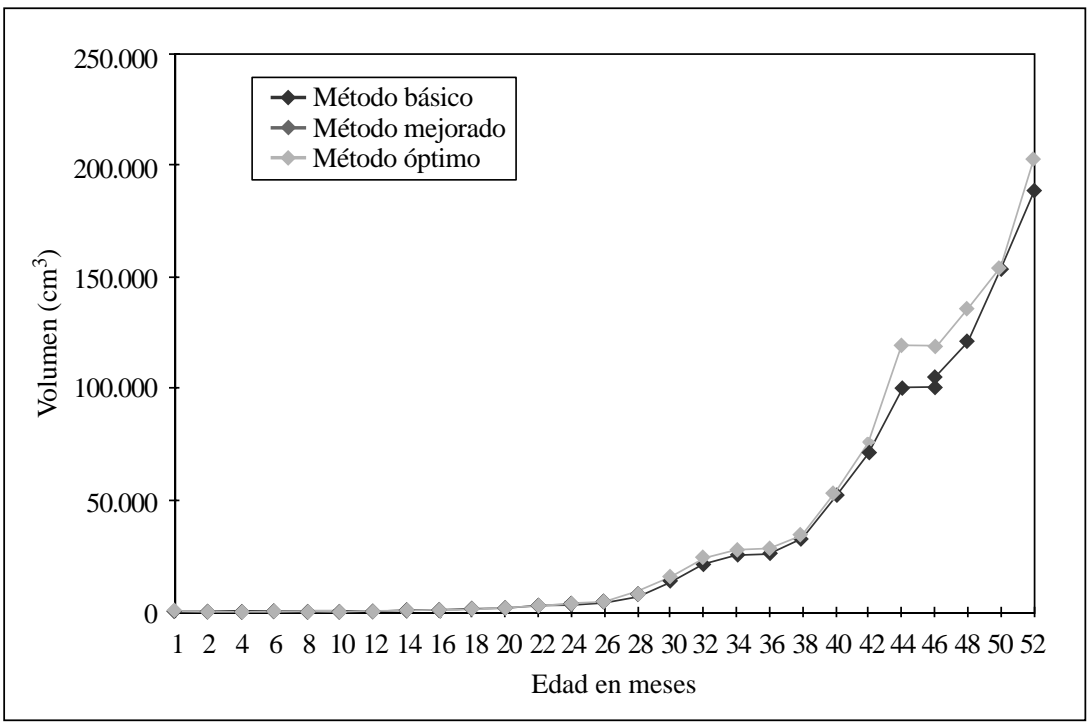

Figura 3. Curvas de crecimiento acumulado para la variable $\mathrm{DAC}^{2 *} \mathrm{H}$. Accumulated $\mathrm{DAC}^{2 *} \mathrm{H}$ index growth curves.

En la figura 4, la variable altura muestra en el crecimiento estacional una dinámica muy parecida en los tres métodos de establecimiento. En todos ellos, la variable altura muestra su punto más alto entre los meses de noviembre y febrero, época que corresponde a la estación de verano. Mientras los puntos más bajos de crecimiento se presentan entre los meses de junio y agosto, época que corresponde a la estación de invierno.

La figura 5, de crecimiento acumulado en altura, muestra muy poca diferencia en el crecimiento de altura entre los métodos de establecimiento.

Si se compara la figura de crecimiento estacional en altura con el diagrama ombrotérmico, se puede identificar una relación directamente pro- 


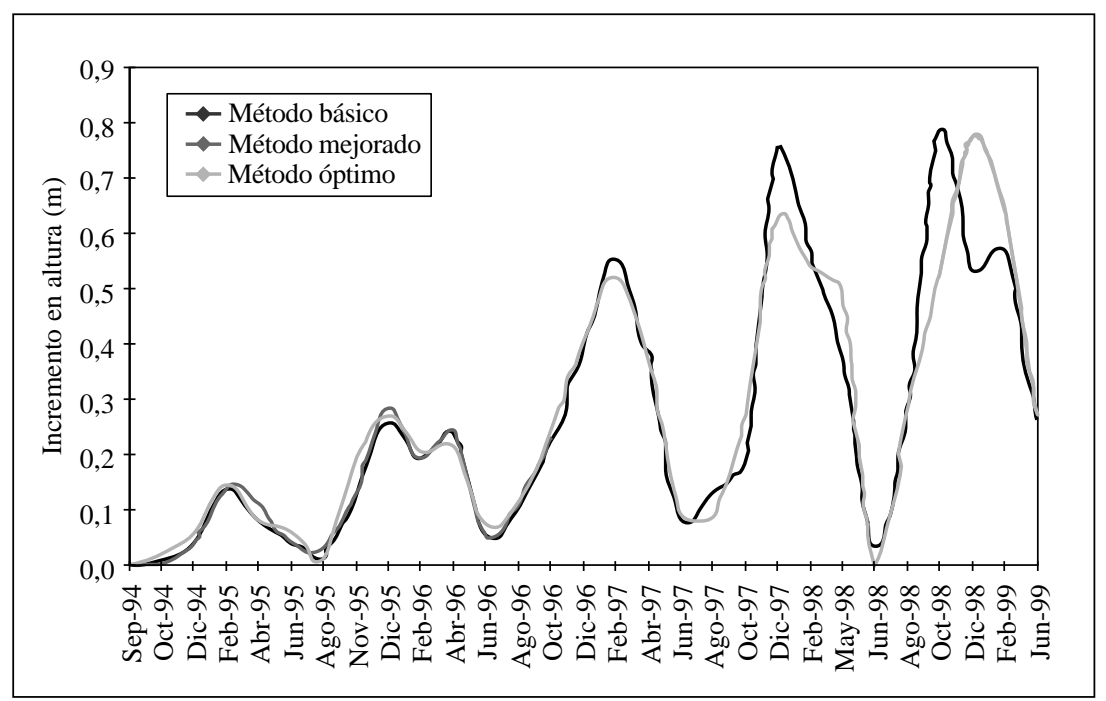

Figura 4. Curva de crecimiento estacional para la variable altura.

Seasonal tree height growth curve.

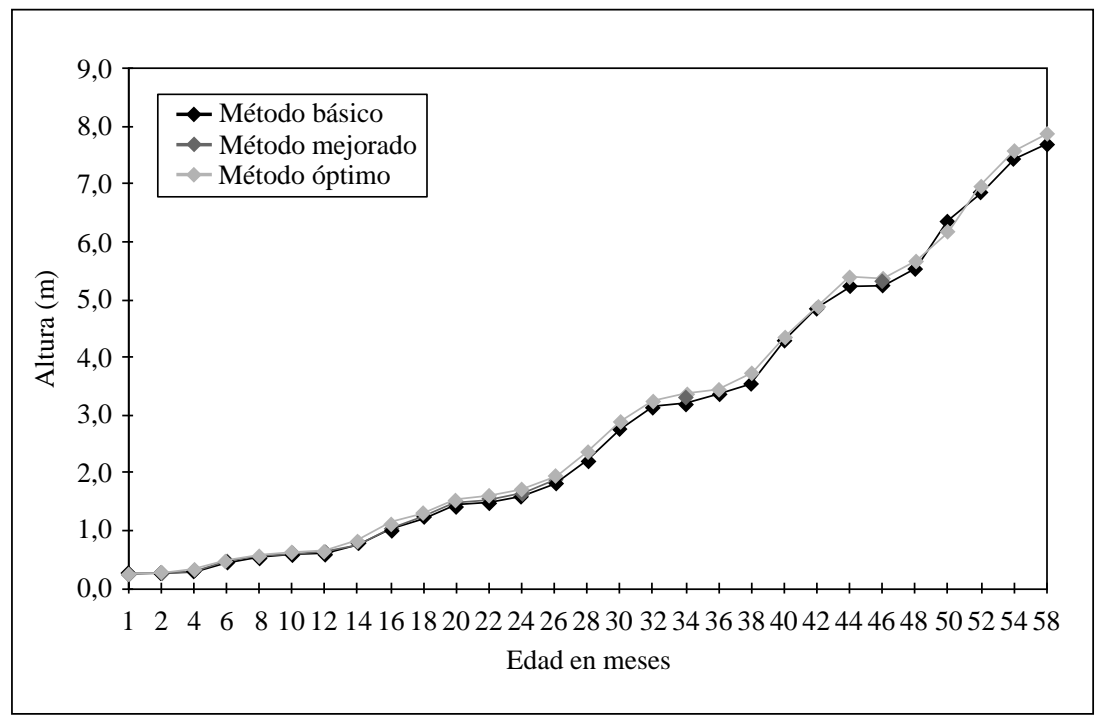

Figura 5. Curva de crecimiento acumulado en altura.

Accumulated height growth curve.

porcional entre las variables de temperatura y el crecimiento estacional. Es decir, en los meses en que la temperatura promedio mensual alcanza valores por encima de $\operatorname{los} 14^{\circ} \mathrm{C}$, el crecimiento en altura es mayor. Mientras que el menor crecimiento se presenta a temperaturas promedio mensuales por debajo de los $10^{\circ} \mathrm{C}$. Lo meses definidos como meses secos no afectan el crecimiento en altura de la especie.
En las figuras 6 y 7 se muestra la curva de crecimiento estacional y acumulado para la variable DAC.

La figura 6, para la curva de crecimiento estacional en DAC, señala tasas de crecimiento más irregulares que las presentadas en el crecimiento de altura. Sin embargo, esta dinámica de crecimiento es común para los tres métodos de establecimiento. En esta condición edafoclimática el 


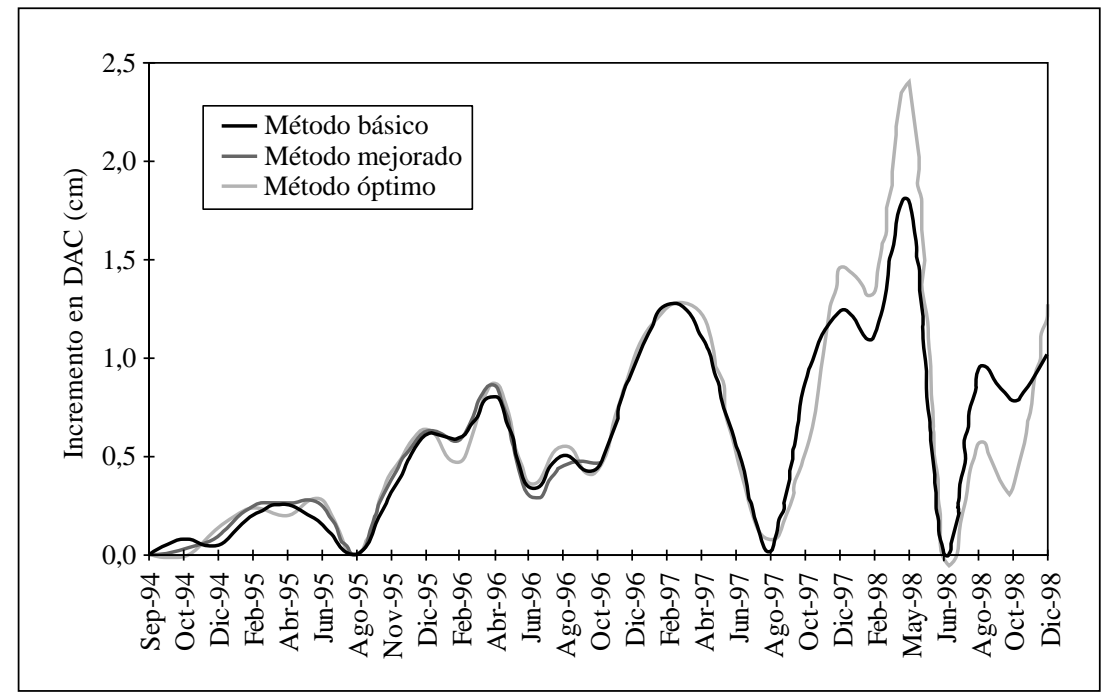

Figura 6. Curva de crecimiento estacional para la variable DAC.

DAC growth curve.

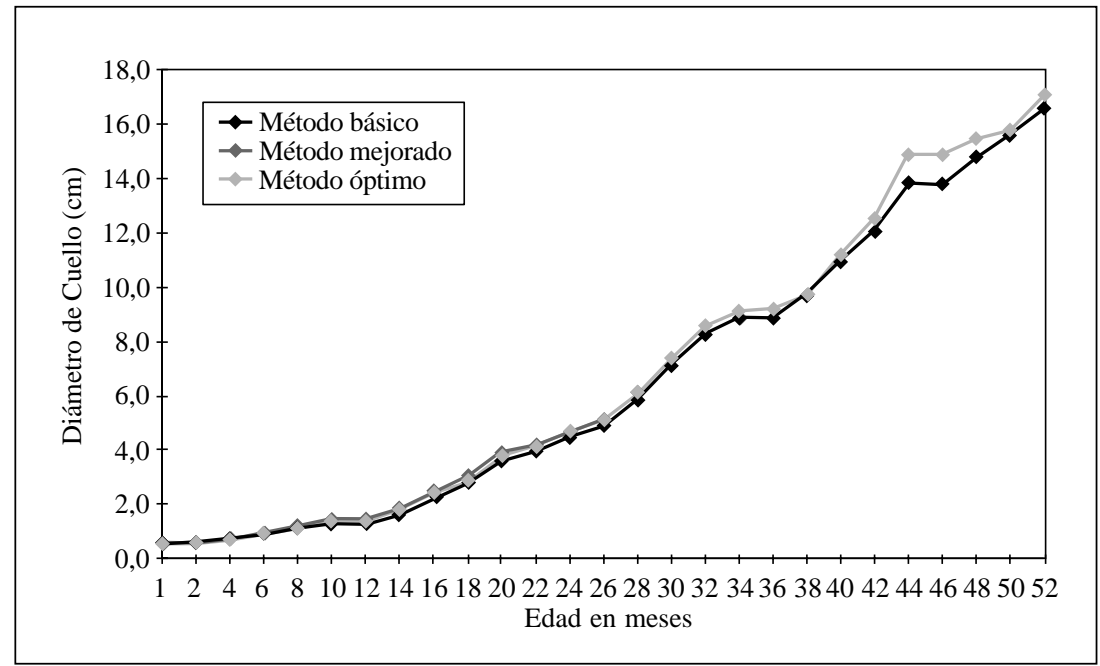

Figura 7. Curva de crecimiento acumulado para la variable DAC.

Acummulated DAC growth curve.

rango de meses cuando se presentan los puntos más altos de crecimiento en DAC es amplio, abarcando de diciembre a mayo. Mientras el menor crecimiento se presenta de junio a agosto. Por otra parte, la figura 7 , de crecimiento acumulado muestra al igual que la altura, muy poca diferencia en el crecimiento de DAC entre los tres métodos de establecimiento.

Al analizar en conjunto los gráficos del crecimiento estacional en DAC con el diagrama om- brotérmico, se puede identificar que tanto los puntos más altos en temperatura (valores promedio mayores a $13^{\circ} \mathrm{C}$ ) como los puntos más altos en precipitación (valores mensuales mayores a 60 $\mathrm{mm}$ ) coinciden con los incrementos en el crecimiento estacional en DAC. Mientras tanto, los puntos más bajos en crecimiento estacional en DAC ocurren en épocas del año donde coinciden bajas temperaturas (menor a $11^{\circ} \mathrm{C}$ ) y baja precipitación (valores mensuales menores a $50 \mathrm{~mm}$ ). 
Al analizar la dinámica de crecimiento de todas las variables estudiadas se identifica concordancia con los estudios realizados por Sandoval (12), Tennet (9), Jackson et al. (7), Aguad (13) y Burdon (10), los cuales sostienen que el crecimiento en diámetro no se comporta de la misma forma que en altura y, por ende, el índice de productividad $\mathrm{DAC}^{2 *} \mathrm{H}$ tiene su propia dinámica de crecimiento. Este último, fuertemente influenciado por el crecimiento en diámetro, ya que éste se encuentra elevado al cuadrado.

Dado lo anterior, el índice $\mathrm{DAC}^{2 *} \mathrm{H}$ se ve fuertemente influenciado por la temperatura y precipitación. El rango de temperatura en el cual se presenta el menor crecimiento concuerda con un estudio citado por Lavery (14), el cual sostiene que el crecimiento diametral del pino radiata continúa durante la mayor parte del año, excepto cuando la disponibilidad de agua es limitada o cuando las temperaturas ambientales son menores a $9^{\circ} \mathrm{C}$. Por otra parte, los períodos de mayor crecimiento concuerdan con Daniels et al. (15), el cual sostiene que el punto óptimo para la fotosíntesis neta está entre los 18 y $25^{\circ} \mathrm{C}$.

\section{- Análisis estadístico del crecimiento}

En primer lugar se presenta el cuadro, 2 que resume los resultados del ANDEVA para cada una de las variables analizadas; posteriormente se interpretan estos resultados.

Para esta situación, el valor F calculado para las variables altura y DAC es menor que el valor
F de tabla. Por lo tanto se concluye, para una probabilidad de error no superior al 5\%, que no existen diferencias estadísticas para estas variables según método de establecimiento. Sin embargo, para la variable $\mathrm{DAC}^{2 *} \mathrm{H}$, sí existen diferencias estadísticas, ya que su $\mathrm{F}$ es mayor.

\section{- Análisis económico}

Los resultados obtenidos en el análisis estadístico del crecimiento para la condición edafoclimática de suelos de "Trumao" reflejan que no existen diferencias significativas según método de establecimiento para las variables DAC y H. Sin embargo, sí las hay, en el crecimiento expresado como índice de productividad. Dado lo anterior, y para simplificar el análisis, se optó por realizar un análisis económico únicamente para el método de establecimiento básico, que representa el menor costo de inversión y resultados satisfactorios de prendimiento y crecimiento.

El primer paso de este análisis fue simular el crecimiento a través del Simulador Radiata Plus Versión 5,2 con las variables de entrada que se presentan en el cuadro 3 .

Con estos antecedentes se obtuvo que el rodal tiene un índice de sitio de 34 metros. Es decir, a los 20 años alcanza una altura media de 34 metros. Dada la alta productividad del sitio se propone un esquema de manejo del rodal en forma intensiva y con el esquema propuesto en el cuadro 4.

Definido el sitio y el esquema de manejo, se determinó el sistema de trozado de los árboles, con los productos que muestra el cuadro 5 .

\section{CUADRO 2}

Andeva para las variables altura, DAC Y $\mathrm{DAC}^{2 *} \mathrm{H}$. ANOVA: Height, DAC and $\mathrm{DAC}^{2 *} \mathrm{H}$.

\begin{tabular}{|l|l|c|c|c|}
\hline $\begin{array}{l}\text { Variable } \\
\text { analizada }\end{array}$ & Fuente de Variación & $\begin{array}{c}\text { Grados } \\
\text { de libertad }\end{array}$ & $\begin{array}{c}\text { Valor F } \\
\text { calculado }\end{array}$ & $\begin{array}{c}\text { Valor F } \\
\text { de tabla }\end{array}$ \\
\hline ALTURA & Métodos de establecimiento & 2 & 1,96 & 5,14 \\
& Error & 6 & & \\
\hline DAC & Métodos de establecimiento & 2 & 4,12 & 5,14 \\
& Error & 6 & & \\
\hline DAC ${ }^{2 * H}$ & Métodos de establecimiento & 2 & 6,10 & 5,14 \\
& Error & 6 & & \\
\hline
\end{tabular}




\section{CUADRO 3}

Datos de entrada del método básico para el simulador.

Entry data for Basic Method simulator.

\begin{tabular}{|l|c|c|c|c|c|}
\hline Fundo & Método & Edad & $\begin{array}{c}\text { Densidad } \\
\text { arb/ha }\end{array}$ & $\begin{array}{c}\text { Área basal } \\
\left(\mathrm{m}^{2} / \mathrm{ha}\right)\end{array}$ & $\begin{array}{c}\text { Altura } \\
\text { dominante }(\mathrm{m})\end{array}$ \\
\hline Santa Lucía & MB & 5 años 10 meses & 1.315 & 25,43 & 10,29 \\
\hline
\end{tabular}

\section{CUADRO 4}

Esquema de manejo intensivo sitio 34.

Intensive management scheme: Site 34.

\begin{tabular}{|l|c|c|c|}
\hline Descripción de podas & Edad & $\begin{array}{c}\text { Densidad } \\
\text { (n/ha) }\end{array}$ & $\begin{array}{r}\text { Largo de copa } \\
\text { remanente }(\mathrm{m})\end{array}$ \\
\hline Poda 1 & 6 & 700 & 4,0 \\
Poda 2 & 7 & 500 & 4,5 \\
Poda 3 & 8 & 500 & 4,5 \\
Poda 4 & 9 & 500 & 4,5 \\
\hline Descripción de raleos & Edad & Densidad remanente (n/ha) \\
Raleo 1 (desecho) & 8 & 600 & \\
Raleo 2 (comercial) & 13 & 400 \\
\hline
\end{tabular}

Fuente: Forestal Mininco S.A. Plan general de manejo 2000. (16).

\section{CUADRO 5}

Esquema de trozado.

Log Cut Scheme.

\begin{tabular}{|l|c|c|c|c|}
\hline Producto & $\begin{array}{c}\text { Diámetro } \\
\text { mínimo }(\mathrm{cm})\end{array}$ & $\begin{array}{c}\text { Diámetro } \\
\text { máximo }(\mathrm{cm})\end{array}$ & $\begin{array}{c}\text { Largo de la } \\
\text { troza }(\mathrm{m})\end{array}$ & Podado \\
\hline Podado 44 a 18 & 44 a 18 & Sin límite & 5,00 & Sí \\
Nudoso 32 a 18 & 32 a 18 & Sin límite & 4,20 & No \\
Pulpable & 10 & Sin límite & 2,44 & No \\
\hline
\end{tabular}

Fuente: Forestal Mininco S.A. Plan general de manejo 2000. (16).

Con los antecedentes expuestos en los cuadros precedentes (cuadro 3, 4 y 5), se obtuvieron los volúmenes totales por producto dentro de un rango de cosecha de 10 a 30 años. Con esta informa- ción, más los datos de costos e ingresos por este método de establecimiento, se realizó el respectivo análisis económico para cada edad de cosecha dentro de un rango de 15 a 25 años (cuadro 6). 


\section{CUADRO 6}

Resultados económicos para el método básico. Nota: US\$1 = \$700.

Basic method economic results. Note: US $\$ 1=\$ 700$ Chilean pesos.

\begin{tabular}{|l|c|c|c|c|c|c|c|c|c|}
\hline Año & $\begin{array}{c}\text { Costos } \\
\text { totales }\end{array}$ & $\begin{array}{c}\text { Ingresos } \\
\text { totales }\end{array}$ & $\begin{array}{c}\text { VAN } \\
8 \%\end{array}$ & $\begin{array}{c}\text { VPS } \\
8 \%\end{array}$ & $\begin{array}{c}\text { VAN } \\
10 \%\end{array}$ & $\begin{array}{c}\text { VPS } \\
10 \%\end{array}$ & $\begin{array}{c}\text { VAN } \\
12 \%\end{array}$ & $\begin{array}{c}\text { VPS } \\
12 \%\end{array}$ & TIR\% \\
\hline & \multicolumn{8}{|c|}{ Datos expresados en miles de pesos chilenos por hectárea } \\
\hline 19 & $6.718,19$ & $11.177,53$ & 116,87 & 136,85 & $-249,60$ & $-274,89$ & $-499,14$ & $-530,34$ & 8,56 \\
20 & $7.170,32$ & $11.992,96$ & 107,64 & 126,04 & $-273,32$ & $-301,11$ & $-527,77$ & $-560,76$ & 8,49 \\
21 & $7.533,26$ & $12.934,32$ & 122,81 & 143,81 & $-279,96$ & $-308,43$ & $-543,94$ & $-577,93$ & 8,53 \\
22 & $7.938,71$ & $13.805,96$ & 107,37 & 125,73 & $-307,17$ & $-338,40$ & $-573,61$ & $-609,46$ & 8,44 \\
23 & $8.300,55$ & $14.715,33$ & 100,25 & 117,39 & $-328,29$ & $-361,67$ & $-598,44$ & $-635,84$ & 8,39 \\
\hline
\end{tabular}

El cuadro 6 muestra que para esta condición edafoclimática, utilizando el método básico de establecimiento, el esquema de manejo "intensivo 34 " y el esquema de trozado propuesto, la actividad forestal es rentable únicamente al utilizar una tasa de actualización del $8 \%$. Además, se puede identificar la edad de 21 años como la edad óptima de cosecha o rotación, debido a que es la edad que presenta el mayor VAN y VPS dentro del rango de edades analizadas.

\section{CONCLUSIONES}

El análisis del crecimiento estacional identificó a través del diagrama ombrotérmico de Gaussen-Walter que los meses secos del año son enero, febrero y marzo. El análisis de las variables de crecimiento altura, DAC y $\mathrm{DAC}^{2 *} \mathrm{H}$ demostró una dinámica de crecimiento a lo largo del año muy parecida entre los tres métodos de establecimiento, pero distinta entre las tres variables. El crecimiento en altura presentó sus valores más altos a temperaturas mayores a $14^{\circ} \mathrm{C}$ (verano), mientras los valores más bajos a temperaturas menores a $10^{\circ} \mathrm{C}$ (invierno). El crecimiento en DAC presenta sus valores más altos a lo largo del año, con temperaturas mayores a $13^{\circ} \mathrm{C}$ y precipitaciones mensuales por encima de los $60 \mathrm{~mm}$ (verano-otoño), mientras el menor crecimiento ocurre a temperaturas menores a $11^{\circ} \mathrm{C}$ y precipitaciones menores a $50 \mathrm{~mm}$ mensuales (invierno). Por último, el máximo crecimiento en $\mathrm{DAC}^{2} * \mathrm{H}$ se presentó a temperaturas alrededor de $\operatorname{los} 12^{\circ} \mathrm{C}$ y precipitaciones mensuales superiores a $100 \mathrm{~mm}$ (otoño).

El análisis estadístico del crecimiento demostró que no existen diferencias estadísticamente significativas al $95 \%$ en el crecimiento, entre los tres métodos de establecimiento (básico, mejorado y óptimo) para la variable de DAC y altura. Sin embargo, para la variable de crecimiento $\mathrm{DAC}^{2 *} \mathrm{H}$, sí se encontraron diferencias estadísticamente significativas entre los tres métodos de establecimiento.

El análisis económico muestra que, al adquirir este tipo de suelo y utilizar el método básico de establecimiento y el esquema de manejo "intensivo 34", la actividad forestal es rentable únicamente a una tasa de actualización de $8 \%$. Al mismo tiempo se identificó al año 21 como la edad en la cual se presentó el mayor VAN al 8\% que fue de \$122.810/ ha; por lo tanto, se definió esta edad como la época de cosecha o rotación.

\section{BIBLIOGRAFIA}

(1) ZWOLINSKI, J., D. SOUTH, L. CUNNINGHAM, S. CHRISTIE. Weed control an large bare-root stock improve early growth of Pinus radiata in South Africa. New Zealand Journal of Forestry Science, 1996, vol. 26, № 1/2, p. 163-172.

(2) MASON, G., D. SOUTH, Z. WEIZHON. Performance of Pinus radiata in relation to seedling grade, weed control, and soil cultivation in the central North Island of New Zealand. New Zealand Journal of Forestry Science, 1996, vol 26, $\mathrm{N}^{\circ} 1 / 2$, p. 173-183.

(3) BALNEAVES, J., M. MENZIES, S. HONG. Establishment practices can improve longer -term growth of Pinus radiata on a dry-land hill forest. New Zealand Journal of Forestry Science. 1996, vol. 26, No 3, p. 370-379. 
(4) STEIN, W. Effectiveness and cost of six site preparation methods in the establishment and growth of Douglas fir. New Zealand Forest Research, 1991 Bulletin No 156. p. 208-214.

(5) DURYEA, M., P. DOUGHERTY. Forest regeneration manual. Kluwer Academic Publishers. Dordrecht, The Netherlands, 1991, Chapter 13.

(6) MACLAREN, J. P. Radiata pine growers manual. New Zealand Forest Research Institute. 1993, Bulletin No 184, p. 17-21.

(7) JACKSON, D. S., H.H. GIFFORD, J. CHITTENDEN. Environmental variables influencing the increment of Pinus radiata: (2) effects of seasonal drought on height and diameter increment. New Zealand Journal of Forestry Science, 1993, vol 3, p. 265-286.

(8) GUTIERREZ, M. P. Estudio del desarrollo vegetativo de árboles jóvenes de Pinus radiata D. Don. Tesis de Ingeniero Forestal. Universidad de Chile. 1977. Santiago, Chile. 85 p.

(9) TENNENT, R.B. Intra-annual growth of young Pinus radiata in New Zealand. New Zealand Journal of Forestry Science, 1986, vol. $16 \mathrm{~N}^{\mathrm{o}}$ 2, p. 166-175.
(10) BURDON, R. D. Annual growth stages for height and diameter in Pinus radiata. New Zealand Journal of Forestry Science, 1994, vol. 24, $\mathrm{N}^{\circ} 1$, p. 11-17.

(11) FORESTAL MININCO S.A. Proyecto Silvicultura Integrada de plantaciones. Departamento de Productividad de Sitios, 2001, Concepción, Chile. 20 p.

(12) SANDOVAL H., E. A. Aplicación de silvicultura integrada en el establecimiento de plantaciones de Pinus radiata D. Don, en suelos de arena de la VIII Región. Tesis de Ingeniero Forestal. Universidad de Chile. 1997. Santiago, Chile. $120 \mathrm{p}$.

(13) AGUAD, F.A. Efecto de la época de poda en el crecimiento de Pinus radiata D. Don. en la precordillera andina de la VII Región. Tesis de Ingeniero Forestal. Universidad de Chile. 1994, Santiago, Chile. 115 p.

(14) LAVERY, P.B. Plantation Forestry with Pinus radiata. Review papers. Paper $N^{\circ} 12$. School of Forestry. University of Canterbury, 1994 Christchurch. New Zealand. 255 p.

(15) DANIEL, T.W., J.A. HELMS, F.S. BACKER. Principios de Silvicultura. Editorial McGraw Hill. 1982, Segunda edición. México. p. 59-70.

(16) FORESTAL MININCO S.A. Plan general de manejo 2000. Informe interno. Concepción 2000, Chile. 25 p. 